\title{
Announcement - Incoming JPET Editor
}

On behalf of ASPET's Publications Committee, I am pleased to announce that Dr. Beverley Greenwood-Van Meerveld has been selected to succeed Dr. Kenneth D. Tew as the next editor of The Journal of Pharmacology and Experimental Therapeutics (JPET). Dr. Greenwood-Van Meerveld will fully assume the editorship in January 2022 when Dr. Tew's term will end.

Dr. Greenwood-Van Meerveld, professor emeritus, has served as George Lynn Cross Research Professor and President's Associates Presidential Professor at University of Oklahoma Health Sciences Center. She was also Senior VA Research Career Scientist at Oklahoma City VA Medical Center.

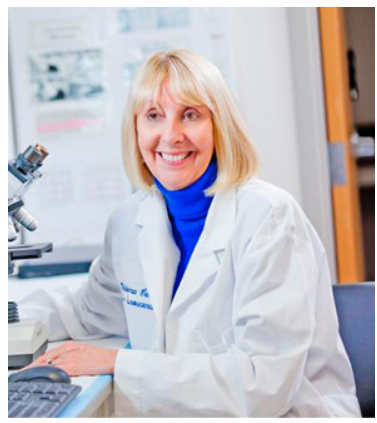

Dr. Greenwood-Van Meerveld is from Northwest England, and after graduating high school in Formby, she received a B.Sc. degree in physiology with special honors from the University of Sheffield, United Kingdom. She received her Ph.D. degree in neurophysiology also from the University of Sheffield in the United Kingdom. Past research and teaching positions include University of Calgary, Medical College of Wisconsin, Eli Lilly and Company, Indiana University, and the Oklahoma Foundation for Digestive Research.

Having recently been selected as an American Society for Pharmacology and Experimental Therapeutics (ASPET) Fellow in 2021, Dr. Greenwood-Van Meerveld has been very active with the Society. She was a member of the ASPET Board of Publications Trustees from 2018 to 2020 and served as a JPET Associate Editor from 2012 to 2018. She has also served on several other editorial boards in various roles, including as an ad hoc journal reviewer since 1986.

Dr. Greenwood-Van Meerveld's primary research interests include the central nervous system regulation of visceral pain with a strategic objective of using preclinical (in vivo and in vitro) rodent models and multiple state-of-the-art techniques, from molecular and cellular to behavioral, pharmacological, and electrophysiological. She studied the neuroanatomical and neuropharmacological substrates involved in the interaction between visceral pain and anxiety. Her team demonstrated long-lasting increases in anxiety-like behavior and viscerosomatic sensitivity in response to stress. Her laboratory found persistent downregulation of glucocorticoid receptor expression and increases in the expression of corticotropin-releasing factor leading to hyperexcitability of central amygdaloid nucleus neurons. Dr. Greenwood-Van Meerveld's research also focuses on understanding sex differences in visceral pain in response to chronic adult stress and various forms of adverse early life stress. Dr. Greenwood-Van Meerveld's laboratory has a long history of engaging pharma to identify novel drug targets to treat visceral pain and disorders, such as irritable bowel syndrome, inflammatory bowel disease, and postoperative ileus.

Her research impact is reflected in 163 research articles, 26 reviews, and 20 editorials, book chapters, and letters. She also holds nine U.S. patents.

Authors interested in publishing in JPET will continue to submit manuscripts through the online manuscript system at https://jpet.msubmit.net/, which will not change with the transition of editors. The Publications Committee is very pleased that Dr. Greenwood-Van Meerveld has accepted the editorship of JPET and looks forward to the continued success of the journal under her leadership.

Dr. Emily E. Scott

Chair 